\title{
Ecología humana y acción pro-ambiental: alteridades recíprocas aula-escuela- comunidad para el manejo sustentable de residuos
}

\section{Human ecology and pro-environmental action: reciprocal otherness amongst the classroom, school and the community for sustainable waste management}

Recibido: $15 / 07 / 2013$

Revisado: 16/09/2013

Aceptado: 03/12/2013

\author{
Javier Guevara Martínez \\ CECA-Granja didáctica El Mezquite, México \\ Departamento de Ciencias Biológicas, UPAEP, México \\ Correspondencia: franciscojavier.guevara@upaep.mx
}

Reconocimientos: La presente investigación se realizó con financiamiento del Consejo Nacional de Ciencia y Tecnología-CONACYT y el Gobierno del Estado de Puebla, Fondos Mixtos (FOMIX 109024). "Proyecto ejecutivo para el manejo integral de los desechos sólidos domésticos en Huauchinango y estudio de caracterización de desechos en cinco zonas urbanas de la Sierra Norte de Puebla”, Javier Guevara responsable técnico. 
the meeting of $42 \%$ of differentiated waste, in a Latin American context of 3 to $6 \%$.

Key words: human ecology, environmental education, elementary school, power environments

\footnotetext{
"La conducta surge en función del intercambio de la persona con el ambiente (entonces); si queremos cambiar las conductas, debemos cambiar los ambientes"
} Urie Bronfenbrenner

\section{La basura como problema de investigación para la Psicología}

El manejo integral de los residuos sólidos aún representa uno de los retos más importantes que enfrentan las autoridades de los gobiernos nacionales, las municipalidades, los prestadores de servicios y la comunidad en general. Los estilos de vida, los altos niveles de consumo, los materiales usados en la producción industrial y la introducción de materiales persistentes en las actividades cotidianas de las personas tienden a incrementar los volúmenes de residuos sólidos. Esto a su vez representa serios problemas para su recolección, transporte, tratamiento y disposición final (Guevara, 2013). En América Latina se producen alrededor de 369.000 toneladas de residuos sólidos municipales diariamente (Stefan, 2010); sin embargo, según una evaluación realizada por la OPS, no se realiza en gran escala la segregación y recuperación de materiales reciclables en América Latina y el Caribe. En promedio, la evaluación mostró que solamente el $2.2 \%$ de los materiales se recupera de la basura, correspondiendo al $1.9 \%$ al reciclaje inorgánico y un $0.3 \%$ al reciclaje de residuos orgánicos (OPS, 2006).

La diversidad de condiciones, señala la OPS, demanda el diseño de "sistemas apropiados" a cada localidad o región, para lograr el objetivo de cobertura o acceso universal a servicios sostenibles y sustentables de manejo de residuos sólidos, lo cual implica procesos de "prueba y error" a través de "proyectos piloto" que conduzcan a su adaptación y mejora continua mediante la innovación en materia de organización, financiamiento y desarrollo de capacidades tecnológicas y socioambientales. Para la OPS (2005), este constituye un tema de estudio que debiera alentarse en los países de América Latina para acelerar el proceso de adopción diferenciados, en un contexto latinoamericano en el que solo se reúne del 3 al $6 \%$.

Palabras clave: ecología humana, educación ambiental, educación básica, entornos de poder

e implementación del nuevo paradigma de prevención y gestión integral de los residuos. Entendido como:

\footnotetext{
... el conjunto articulado e interrelacionado de acciones normativas, operativas, financieras, de planeación, administrativas, sociales, educativas, de monitoreo, supervisión y evaluación, para el manejo de residuos, desde su generación hasta la disposición final, a fin de lograr beneficios ambientales, la optimización económica de su manejo y su aceptación social, respondiendo a las necesidades y circunstancias de cada localidad o región.
}

No hay elementos que indiquen que la región transite en esa dirección; las políticas públicas han hecho depender el manejo de la basura a factores técnicos y escasa o nulamente a su componente social (Guevara \& Rodríguez, 2003). Lo anterior se expresa en múltiples experiencias exitosas en nuevas rutas y mecanismos de recolección y, aún con déficit, en los nuevos centros de confinamiento de los residuos, pero sin programas orientados a la población.

Tabla 1

Ruta actual de residuos sólidos municipales.

\begin{tabular}{ccc}
\hline \multicolumn{3}{c}{ Ruta actual de residuos sólidos municipales } \\
\hline FASE 1 & FASE 2 & FASE 3 \\
\hline Generación & Recolección y Traslado & Destino Final \\
\hline $\begin{array}{l}\text { Reunión día a día de } \\
\text { residuos producidos } \\
\text { en casa }\end{array}$ & $\begin{array}{c}\text { Sistema municipal de } \\
\text { recolección }\end{array}$ & $\begin{array}{c}\text { Depósito de residuos } \\
\text { en el Relleno sanitario }\end{array}$ \\
\hline $\begin{array}{l}\text { Las políticas públicas han realizado un gasto permanente para las etapas DOS } \\
\text { y TRES, adquiriendo vehículos de carga y transportación, y para construir }\end{array}$ \\
complejos sitios de confinamiento para sepultar los residuos.
\end{tabular}

La meta más importante para el manejo sustentable de residuos, es el confinamiento separado para su manejo diferenciado, así que, sin abandonar los avances logrados hasta el momento, proponemos atención especial a la primera fase, la de generación de residuos, soportando en la participación ciudadana la solución principal al problema. Cabe señalar que la conducta de separación de residuos ha sido una de las más estudiadas dentro de la Psicología 
Ambiental, por ser considerada una de las conductas proambientales más importantes (Durán, Alzate \& Sabucedo, 2009, p. 28).

Basar la estrategia en la participación social, significa atacar el problema en su origen, lo cual implica cambiar la cultura predominante. La operacionalización de la "cultura de la basura" implica cuando menos una esfera conductual y una cognitiva; así, cuando nos proponemos incidir para cambiarla por una cultura de acopio y entrega diferenciada de residuos, en realidad hablamos de la generación de una conducta ecológicamente relevante o proambiental, y de una estructura sociocognitiva proclive al ambiente.

Prácticamente todos los educadores y/o ambientalistas que participan en procesos similares se refieren a la colecta selectiva, implícita referencia al papel gubernamental en el proceso. Esto omite la otra parte, la responsabilidad ciudadana, así que preferimos el concepto de entrega diferenciada (concepto complementario a colecta selectiva que pone énfasis en la participación social). Así, la Educación Ambiental deberá procurar su "integración a la sociedad en torno a la generación y articulación de propuestas que busquen la consolidación de una cultura ambiental en el planeta, que atienda las emergencias que demanda la sociedad actual en la transformación de las relaciones de los individuos con el entorno" (Flores, 2012, p. 94).

\section{Contexto y sentido de la intervención}

Con múltiples financiamientos gubernamentales e intervenciones a diversas escalas, se ha desarrollado el Modelo de Intervención Socioambiental para el Manejo Integral de Residuos, participando psicólogos (sociales, ambientales, educativos y comunitarios) ingenieros ambientales (caracterización de residuos); economistas (estudio de mercado, economía solidaria); cientistas de la comunicación (estrategias en medios); pedagogos (procesos educativos); ingenieros (rutas de traslado y centros de acopio de residuos) y agro-biólogos (compostaje).

Más que la suma de disciplinas, el presente estudio intenta asir, bajo saberes disímiles, el polígono de intervención como unidad compleja. La idea de unidad compleja, dice Morin (2008, p. 135) adquiere densidad si asumimos que "no podemos reducir ni el todo a las partes, ni las partes al todo; ni uno al múltiplo, ni el múltiplo a uno, pero que necesitamos intentar concebir en conjunto, de modo complementario y antagónico, las nociones del todo y de las partes, de uno y de diversos". En consecuencia, la educación ambiental que propongo en la escuela (EAF), tiene como punto de partida el concepto de Ecosistema, “......una representación del Todo, tomado como sistema, de la misma forma en que el término fue tomado prestado de las concepciones que lo fundaran: el holismo y la teoría de sistemas (Pontes, 2013, p. 20), siendo considerado el concepto de diversidad cultural como un componente integral de la biodiversidad (Alegre, 2007). Así, la investigación que se reporta se vertebra meta-teóricamente desde la ecología humana ${ }^{1}$ para así lograr la emergencia de una cultura de separación y entrega diferenciada de residuos.

Como observa Lewin (1936), no es fácil cambiar culturas. Uno de los obstáculos interpuestos a ese cambio, y que-afirma el autor-debe tenerse en cuenta "es que es un proceso en un campo estándar que no se refiere sólo a esos aspectos". En realidad, dicho estándar se encuentra interrelacionado con todos los aspectos de la cultura (Damergian, 2013). El concepto abarca, tanto la conducta molar del individuo o individuos, como las características físicas del contexto concreto donde se produce esa conducta. Así que para generar un cambio significativo, tiene que darse una determinada interrelación entre los entornos, componentes que interactúan entre sí, de modo que dan lugar a programas diferentes según sean unos u otros esos componentes.

Urie Bronfenbrenner (1987) recogerá en su teoría de los escenarios del desarrollo humano la tradición fenomenológica y social de Lewin, proponiendo la ecología del desarrollo humano como vía integradora para el estudio del individuo en desarrollo y, la conceptualización del entorno en estructuras concéntricas, a las que denominará micro-meso-exo y macrosistemas que afectan al sujeto de forma diferente.

El comportamiento humano no puede explicarse adecuadamente si aislamos a las personas de los entornos en que se desenvuelven, la conducta de los individuos varía en función de aspectos concretos de la cultura donde ocurren, es decir, de la situación en la que se desarrolla la tarea. Los

1 Aunque el objetivo de la investigación se orienta a uno de los problemas ambientales más graves, guardo distancia de la utilización del prefijo "eco-" como referencia al ambiente natural, "que proviene de un equívoco: el uso del vocablo ecología como sinónimo de ambiente natural por los medios norteamericanos, a comienzos de los ańos 60" (Golley, 1993, p. 221, citado por Pontes, op cit). 
contextos, sin embargo, no están dados sino que se constituyen mutuamente y están en constante cambio; las definiciones de las situaciones se logran con el trabajo y la interacción de los participantes. Así, los contextos son marcos culturales de interpretación, lo que modifica la idea de la cultura como guión para producir ocasiones sociales, reestructurando los guiones culturales como conjuntos de principios para la construcción de hechos sociales. Quizá, afirman Rojas y Lara (2004) co-construcción sea un término más adecuado.

Para inducir un proceso de implicación de la escuela y la comunidad en un mismo plano de acción, lo que se requiere no es sólo una relación complementaria entre los campos, sino su integración funcional (Bronfenbrenner, op. cit. p30). Es así porque la educación no se limita a la educación escolar, ni los aprendizajes necesarios para la vida, para el trabajo, para la participación, para la ciudadanía plena pueden limitarse a un período determinado de la vida de una persona. El aprendizaje se inicia con el nacimiento y se extiende a lo largo de toda la vida, arranca en el hogar, antecede y excede a la institución escolar, abarcando un amplio conjunto de instituciones, modalidades, relaciones y prácticas. La educación, la comunidad educativa y la política educativa son mucho más amplias, respectivamente, que la educación escolar, la comunidad escolar y la política escolar (Torres, 2001).

\section{Método}

\section{Diseño}

El eje de la intervención es la alteridad que ocurre entre un entorno o campo psicológico y otro, así como las múltiples formas en que las interacciones (siempre alteridades) entre los entornos (micro-meso-exo y macro) producen fenómenos culturales, formas sociales o modos y estilos de vida. Lewin (1936) atribuye a un campo psicológico:

a) la fuerza, que expresa la tendencia a actuar en una cierta dirección;

b) la posición, que se refiere al estatus de la persona o entidad colectiva respecto de otras que están en ese campo;

c) la potencia, que se relaciona con el peso que una cierta área del campo tiene para la persona y los colectivos en relación con otros.

El establecer estas relaciones, genera y potencia "la sinergia que existe entre los diferentes elementos que componen el sistema ambiental, y cómo cada acción que se realiza sobre un elemento del sistema, influye en otros" (Alegre, 2010, p.5).

La propuesta metodológica considera la estrategia de los experimentos ecológicos lewinianos para investigar empíricamente las interdependencias de los contextos. Esta teoría no se enfoca únicamente al ambiente y al sujeto que lo habita, sino a las interconexiones ambientales y su impacto sobre las fuerzas que afectan directamente el desarrollo psicológico, por esta razón los sujetos son los profesores y estudiantes agrupados en sus entornos naturales.

\section{Participantes}

La población de la "macro-intervención" son los 51,000 habitantes de la ciudad de Huauchinango y las múltiples entidades sociales actuantes en éste polígono de estudio, una de ellas, la escuela.

Para el programa de EAF, la investigación interviene en el cien por ciento de la población educativa y los sujetos colectivos, son los 397 grupos académicos en las 60 escuelas de la ciudad, reuniendo a una cantidad de 397 profesoras(es) y 8266 escolares, distribuidos como se presentan en la tabla 2.

Tabla 2

Distribución de los participantes.

\begin{tabular}{lccc}
\hline \multicolumn{4}{c}{ Distribución de los participantes } \\
\hline Nivel & №escuelas & № profesores & № alumnos \\
\hline Preescolar & 36 & 138 & 1007 \\
\hline Primaria & 24 & 259 & 7259 \\
\hline Total & 60 & 397 & 8266 \\
\hline
\end{tabular}

\section{Instrumentos}

Son tres los instrumentos: dos de carácter evaluativo, y uno substancial-radical ${ }^{2}$. Los primeros son el diferencial semántico o Escala de Ossgod, para reportar los cambios

2 Los instrumentos profundos en la intervención son los movimientos vivos y las interacciones entre los entornos, así que lo substancialradical se refiere a la profundidad del proceso (en el sentido de raíz). La complejidad de la investigación llevó a la elaboración de tres reportes del proceso de EAF. Se publicará en breve "Alteridad de entornos" con la doctorante Thalía Ceja y "La tarea como vínculo", con el Dr. Ignacio Ibarra, donde el énfasis son las variables e indicadores del proceso. 
en las actitudes de los ciudadanos, referente a "servicio de recolección de basura" y, referente a "separación de basura"; y la observación directa, para reportar el comportamiento proambiental colectivo (o acopio y entrega diferenciada de residuos), mientras que el tercero se refiere a los procesos de interconexión ambiental entre los entornos, para posicionar un comportamiento proambiental en la población. Es un instrumento de cambio.

Diferencial semántico o Escala de Ossgod. El interés de una investigación aplicada es la solución del problema, en este caso la cooptación de altos volúmenes de residuos separados, provenientes de la acción ciudadana, por esta razón se evalúan las actitudes de la población abierta, para saber cuáles fueron los cambios subjetivos en la población, que son los habitantes de la ciudad.

Se aplicó una adaptación a la Escala de Ossgod o diferencial semántico reportado hace más de una década, en aquel momento para construir el instrumento y reportar el cuerpo actitudinal (pre-test) de la población objetivo, según sus atributos demográficos; en una población experimental de ocho mil ciudadanos, para localizar sus actitudes (enfatizando el cuerpo principal de creencias) con relación a la "separación de la basura doméstica", y con respecto al "servicio de recolección de basura” (Guevara \& Rodríguez, 2002).

Con el mismo instrumento se reportó un año después la evaluación de los cambios actitudinales de la población objetivo. Dicho reporte contrasta los resultados de la evaluación pretest con la postest para estimar el cambio actitudinal generado por el modelo de intervención (Guevara \& Rodríguez, 2003), en ese caso, con énfasis en la comunicación persuasiva o, según la discusión en el presente reporte, de la EA Informal.

En el presente reporte, igual que en aquellos, se replicó el procedimiento con los estímulos "basura" y "servicio de recolección de basura", con veinte polaridades cada uno; con 459 participantes en la fase pretest y con 454 en postest (Quintanar, 2011). La medición de las actitudes antes y después de la intervención tiene ahora un papel subsidiario, es decir, más que los mecanismos de cambio, interesa saber si hubo cambio.

Observación directa. La observación se realizó en 57 barrios de la ciudad de Huauchinango, al término de la segunda etapa (de cuatro). Consistió en el registro simple de la entrega de los residuos diferenciados (orgánicos, reciclables y misceláneos), reportando el número de familias en los polígonos barriales, y por tanto el porcentaje de residuos diferenciados en cada barrio.

Instrumento substancial-radical. De los múltiples entornos en los que se despliega la vida del escolar, se interviene estratégicamente en tres, uno de ellos de extensión educativa - por tanto fuera de la escuela - que alcanzan el rol de instrumento a partir de sus interconexiones ambientales. Por esta razón, el interés metodológico son los entornos de poder en la relación del estudiante con su realidad, asumiendo que "el potencial evolutivo de un entorno varía en relación inversa al número de vínculos intermedios en la cadena de la red que conecta ese entorno con los entornos de poder" (Op. cit., p. 280). Esto es, la conexión directa entre la sesión académica sobre los residuos (entorno aula), con la cobertura, coherencia y consistencia de su institución (entorno escuela) y la vida social significativa del escolar (entorno familia-comunidad), convierte a cada uno de ellos en entornos de apoyo para el cambio cultural, bajo un proceso de alteridad recíproca, aunque asimétrica.

En la perspectiva de la ecología humana, en primer lugar, el desarrollo supone un cambio en las características de la persona que no es efímero ni depende de la situación; implica una reorganización que tiene una cierta continuidad tanto en el tiempo como en el espacio. En segundo lugar, el cambio de desarrollo tiene lugar, a la vez, en dos campos: el de la percepción y el de la acción. En tercer lugar, desde un punto de vista teórico, cada uno de estos campos tiene una estructura que es isomórfica con los cuatro niveles del ambiente ecológico.

\footnotetext{
"en la esfera de la percepción, la cuestión pasa a ser en qué medida el punto de vista que tiene del mundo la persona en desarrollo se extiende más allá de la situación inmediata, para incluir una imagen de otros entornos en los que ha participado activamente, las relaciones entre éstos, la naturaleza y la influencia de los contextos externos con los que no ha tenido un contacto cara a cara, y, por último, los patrones coherentes de organización social, sistemas de creencias y estilos de vida, que son específicos de su propia cultura" (Op. cit., p47).
}

La actividad de manejo sustentable de residuos es entendida como actividad molar. Éstas, las actividades molares son formas de conducta, pero no todas las conductas son formas de actividad molar. Una actividad molar es una conducta progresiva que posee un momento propio, y que tiene un significado o una intención para los que participan en el entorno. 
Los términos molar y progresiva se emplean para destacar que una actividad es más que un hecho momentáneo, como un movimiento o una expresión oral; por el contrario, es un proceso continuo que requiere algo más que un principio o un fin. Otra propiedad, aún más distintiva, de las actividades molares, que Lewin (1936) destaca, es el hecho de que se caracterizan por tener un momento propio, un sistema de tensión, que contribuye a su persistencia en el tiempo y a su resistencia a la interrupción hasta que se completa la actividad. En su mayor parte, este momento se produce por la existencia de intención.

Son tres los entornos de intervención:

1. Entorno aula. Para construir las estrategias didácticas se realizó un diagnóstico de contexto, también se hizo revisión curricular para conocer los componentes temáticos de educación ambiental incluidos en los programas. El eje del entorno aula son cinco estrategias didácticas, organizadas en dos bloques: el primero de problematización en el campo afectivo y cognitivo, con tres estrategias (magnitud, efectos sanitarios, estéticos, financieros de la basura); y el segundo bloque, comportamental, con dos estrategias experienciales y vivenciales que buscan un "nivel - puntualde desempeño alcanzado por los estudiantes" Torres, Mora, Garzón y Ceballos (2013 p. 195). La primera estrategia (del segundo bloque), que se realiza en el aula y que da como tarea la separación de residuos en casa, y la segunda, que capacita al estudiante para persuadir a vecinos, familiares y/o amigos para la separación.

Los (as) profesoras(es) son capacitados (as) previamente para la aplicación de las estrategias didácticas que "vincule la praxis docente con la teoría vigente" (Feo, 2010, p 227) que incluye la realización de separación de residuos en casa (no se puede persuadir a alguien, de lo que no se está persuadido), muestreando para la observación del cumplimiento de la tarea durante el periodo de capacitación.

2. Entorno Escuela. Tiene el propósito de convertirlo en un entorno con dinámica propia, vinculado directamente al proceso de manejo de residuos, generando a su vez los factores contextuales para convertirlo en un entorno de apoyo al entorno aula.

Se definen las variables que concretan el vínculo, enfatizando las actividades escolares cotidianas, así como los actores intervinientes, como padres de familia, cuerpo directivo, etc.

3. Entorno comunidad. En el macro-entorno comunidad y los exo-entorno(s), participan actores diversos (iglesia, deportistas, grupos territoriales, instituciones, etc.), en los cuales también se despliegan estrategias, algunas de ellas, como la animación sociocultural y la comunicación en medios masivos, que dan omnipresencia al problema basura, permeando al conjunto de la población.

La escuela "está llamada a responder y debe actuar como agente de cambio, fomentando la vida comunitaria y privilegiando las familias" (Fuenmayor, 2008, p. 56), por esta razón participa estratégicamente en cada uno de los momentos de la intervención, articulada a las acciones generadas en otras entidades. Y, aunque "la actual publicidad está implementando nuevas formas persuasivas que no se perciben" (Bermejo, 2013), son muy pocas las influencias externas que afectan de una manera significativa la conducta y el desarrollo humano, que pueden describirse exclusivamente en función de las condiciones y los hechos físicos objetivos; "los aspectos del ambiente que modelan con más fuerza el curso del desarrollo psicológico son, sin duda, aquellos que tienen significado para la persona en una situación determinada" (Bronfenbrenner, p 41).

La tabla 3 resume los componentes de cada uno de los entornos, presentados como categorías, desagregando las variables e indicadores en cada una de ellas:

\section{Procedimiento}

Metodológicamente, la intervención en la escuela se articula con las fases y etapas del Modelo de intervención; se apoya en las estrategias psicosociales de influencia social y comunicación persuasiva, para inducir la cultura emergente desde entornos específicos, induciéndolos como entornos de apoyo (siempre recíprocos).

Las estrategias "permiten involucrar a estudiantes, padres y representantes, entre otros miembros, que discutan, estudien y analicen temas de la Educación Ambiental" (Ramírez, 2008, p. 108). Así, los maestros deben ver a la familia como su mejor aliado en el proceso de enseńanza y de aprendizaje y viceversa. La comunidad, por su parte, es el contexto donde se pueden enriquecer los procesos de enseñanza y de aprendizaje, a su vez, puede junto a la escuela realizar 
Tabla 3

Codificación de segmentos.

\begin{tabular}{|c|c|c|}
\hline \multicolumn{3}{|c|}{$\begin{array}{l}\text { Desagregación de componentes para el comportamiento } \\
\text { molar de los escolares }\end{array}$} \\
\hline Categoría & Variable & Indicador \\
\hline \multirow{6}{*}{$\begin{array}{l}\text { Entorno } \\
\text { AULA }\end{array}$} & Profesora (or) & Asistió a capacitación \\
\hline & $\begin{array}{l}\text { Capacitación- } \\
\text { entrenamiento }\end{array}$ & $\begin{array}{l}\text { Fue capacitado(a) por } \\
\text { facilitador(a) }\end{array}$ \\
\hline & & No tuvo capacitación \\
\hline & Estrategias didácticas & Se aplicaron 5 estrategias \\
\hline & & Se aplicaron menos de tres \\
\hline & & Se aplicó estrategia 4 y 5 \\
\hline \multirow{6}{*}{$\begin{array}{l}\text { Entorno } \\
\text { ESCUELA }\end{array}$} & $\begin{array}{l}\text { Proceso de asimilación } \\
\text { institucional del } \\
\text { programa }\end{array}$ & $\begin{array}{l}\text { Reunión y acuerdo de academia } \\
\text { Seguimiento de acuerdos } \\
\text { Plan institucional de trabajo }\end{array}$ \\
\hline & Acción institucional & Comisión de ecología \\
\hline & & Patrulla de limpieza \\
\hline & & Periódico mural \\
\hline & & Participación de padres \\
\hline & & Ceremonias \\
\hline \multirow{12}{*}{$\begin{array}{l}\text { Entorno } \\
\text { COMUNIDAD }\end{array}$} & NO separar residuos & No puede, trabaja \\
\hline & & No puede, no llega camión \\
\hline & $\begin{array}{l}\text { Separar los residuos } \\
\text { propios }\end{array}$ & $\begin{array}{l}\text { No quiere, desconfía del } \\
\text { gobierno }\end{array}$ \\
\hline & & No quiere, no sirve \\
\hline & & Separar durante un tiempo (hasta \\
\hline & & 2 meses) \\
\hline & & Separar permanentemente con \\
\hline & & errores \\
\hline & & $\begin{array}{l}\text { Separar adecuada y } \\
\text { permanentemente }\end{array}$ \\
\hline & Persuasión & Persuadir a una familia \\
\hline & Pro-ambiental realizada & Persuadir a más de una familia \\
\hline & & Realizar actividades adicionales \\
\hline
\end{tabular}

proyectos de índole social que permitirá un desarrollo para ambos componentes (Fernández y Bigott, 2011, p. 90).

No hay que olvidar que para la ecología humana la capacidad de un entorno para funcionar de manera eficaz (como contexto para el desarrollo) depende de la existencia y la naturaleza de las interconexiones sociales entre los entornos. Esto incluye la participación conjunta, la comunicación y la existencia de información en cada entorno con respecto al otro (Bronfenbrenner, 1987, p. 25).

La intervención en los entornos se hace bien sea de manera simultánea, o bien en distintos momentos
Tabla 4

Etapas de la investigación (y de la escuela) y desagregación de variables (acciones).

\begin{tabular}{|c|c|c|c|}
\hline $\begin{array}{c}\text { Fases en la } \\
\text { escuela }\end{array}$ & $\begin{array}{c}\text { (Fases) } \\
\text { Acciones en la } \\
\text { Comunidad }\end{array}$ & $\begin{array}{l}\text { Acciones en } \\
\text { el ENTORNO } \\
\text { ESCUELA }\end{array}$ & $\begin{array}{c}\text { Acciones en } \\
\text { el ENTORNO } \\
\text { AULA }\end{array}$ \\
\hline $\begin{array}{l}\text { M1 } \\
\text { Disposición }\end{array}$ & $\begin{array}{l}\text { Análisis del } \\
\text { modo de vida }\end{array}$ & $\begin{array}{l}\text { - Acuerdo con } \\
\text { padres de } \\
\text { familia } \\
\text { - Comisión de } \\
\text { ecología. }\end{array}$ & $\begin{array}{l}\text { Capacitación de } \\
\text { cuerpo docente }\end{array}$ \\
\hline $\begin{array}{l}\text { M2 } \\
\text { Acceso }\end{array}$ & $\begin{array}{l}\text { Intervención en } \\
\text { la estructura } \\
\text { social de la } \\
\text { comunidad }\end{array}$ & $\begin{array}{l}\text { - Ceremonia(s } \\
\text { Ex profeso } \\
\text { - Periódico } \\
\text { mural. } \\
\text { - Patrulla de } \\
\text { limpieza } \\
\text { - Selección } \\
\text { residuos } \\
\text { (docentes) }\end{array}$ & $\begin{array}{l}\text { Aplicación de } \\
\text { estrategias } \\
\text { didácticas } \\
\text { (simultánea en } \\
\text { todos los grupos } \\
\text { académicos) }\end{array}$ \\
\hline $\begin{array}{l}\text { M3 } \\
\text { Despliegue }\end{array}$ & $\begin{array}{l}\text { Influencia social } \\
\text { orientada a la } \\
\text { causa ambiental }\end{array}$ & 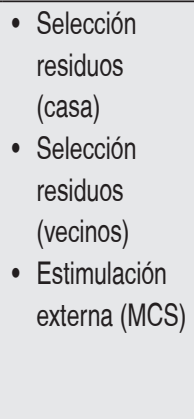 & $\begin{array}{l}\text { Acción directa } \\
\text { en casa: } \\
\text { Participación de } \\
\text { la familia. } \\
\text { Acción } \\
\text { comunitaria: } \\
\text { Participación de } \\
\text { familia extensa, } \\
\text { amigos y/o } \\
\text { vecinos }\end{array}$ \\
\hline
\end{tabular}

pero siempre articulados. La investigación cubre dichos momentos, a través de diversas acciones.

La escuela se define entonces como institución comprometida con el derecho a educarse, y por ello genera las condiciones que hagan factible una distribución equitativa de bienes simbólicos (culturales, sociales, pedagógicos) y el fortalecimiento de las condiciones materiales, definidas en diálogo con la comunidad que constituye su contexto (Giacobbe \& Moscoloni, 2009, p. 5).

Hay, simultáneamente a las estrategias dentro de la escuela, estrategias fuera de ella, inducida a través de los escolares. Las prácticas de la educación no formal se desarrollan usualmente como prácticas extramuros escolares, en las organizaciones sociales, en los movimientos (Ghon, 2009). 


\section{Resultados}

\section{Actitudes orientadas a "basura" y "servicio de recolección de basura"}

El análisis de resultados de la presente investigación se centró en dos aspectos principales; por un lado se presentan dos gráficas para cada uno de los estímulos evaluados con el diferencial semántico por separado y con veinte polaridades cada uno; en este caso se presentan la suma total de los valores obtenidos en cada una de las polaridades del diferencial semántico con 459 participantes en la fase pretest y con 454 en postest. En el caso de los dos estímulos (Separar Basura y Servicio de recolección de Basura) se evaluaron con una serie de 20 polaridades cada uno, que se ponderaban de -3 para lo menos favorable a +3 como lo más favorable.
En la figura 1 se muestran los resultados para el estímulo Separa Basura, se observa que de las 20 polaridades solamente cuatro presentan valores pretest inferiores al postest, es decir que pasaron de desconocido a algo más conocido, de indiferencia a interés, de desagradable a agradable y de lento a rápido. En el resto de las polaridades los valores pretest fueron ligeramente superiores al postest. Sin embargo, en el caso de las polaridades bueno-malo, costoso-barato, insignificante-importante, inteligente-tonto, indecisodecidido se obtuvieron las puntuaciones más altas.

En la figura 2 se tienen las polaridades para el estímulo Servicio de Recolección de Basura, y se puede observar que los valores tienen mayor variación que en el caso de la figura 1. pero en el caso de la quinta polaridad, referente

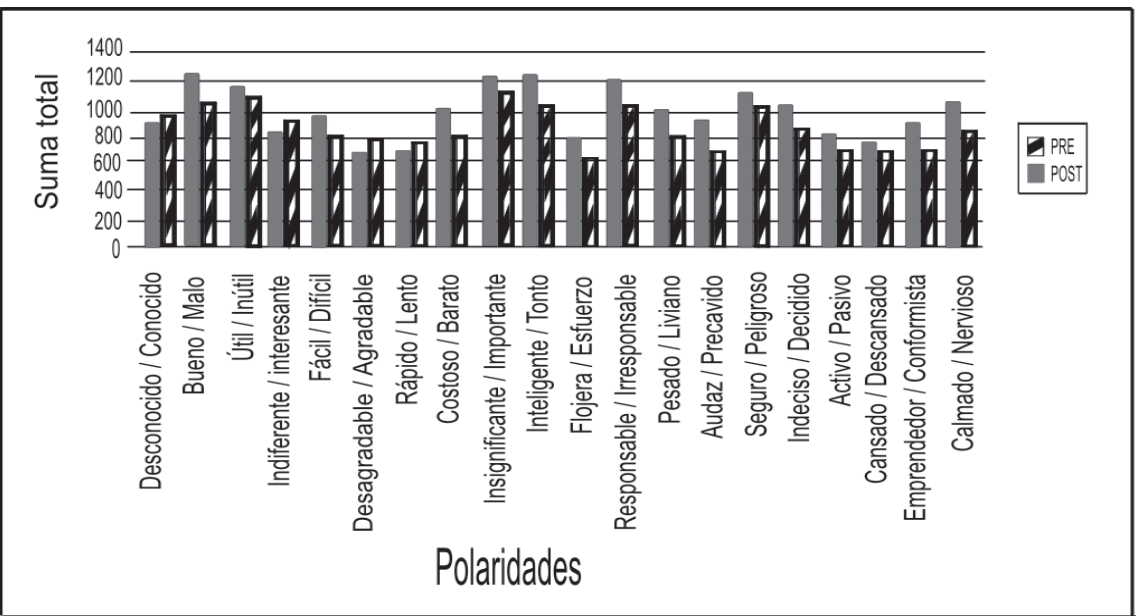

Figura 1. Puntuaciones totales pre y post del diferencial semántico para el estímulo.

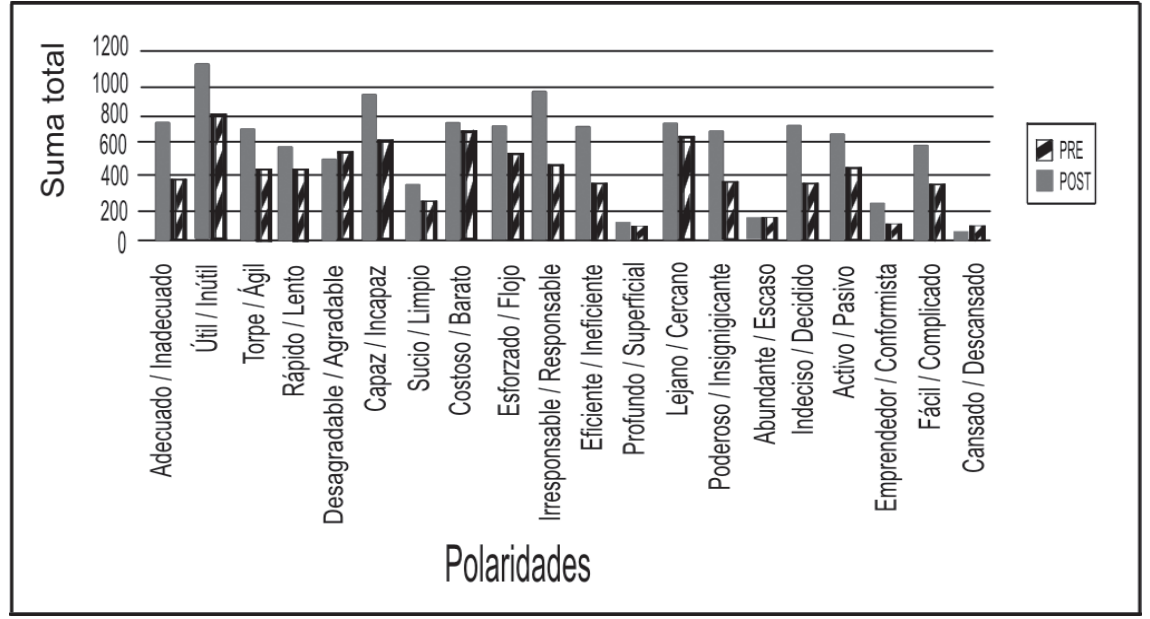

Figura 2. Puntuaciones totales pre y post del diferencial semántico para el estímulo servicio de recolección de basura. 
a desagradable-agradable, se tiene el único caso donde se observa una inversión de los valores donde el pretest es ligeramente mayor que el postest. La segunda polaridad (útil-inútil) y la sexta (capaz-incapaz) son las que tuvieron los valores más altos en el pretest, y tuvieron una disminución notable en el postest, que a pesar de ser algo positivo no tiene el mismo valor que se le atribuía al principio. Las polaridades referentes a profundo-superficial, abundanteescaso y cansado descansado tienen los valores menores en relación a las otras polaridades; pero en la última polaridad cansado-descansado se observó un ligero aumento de pretest a postest es decir, se valoró mejor el servicio de recolección al pasar de cansado a descansado.

También es importante notar que en general el segundo estímulo no tuvo una valoración tan alta como el primero en la mayoría de las polaridades.

\section{Comportamiento ciudadano proambiental}

El Servicio de Recolección transitó por las rutas habituales en los barrios de la ciudad, registrando durante siete días consecutivos el tipo de entrega: diferenciada o no diferenciada, de los residuos del universo total de la población.

Hasta la segunda etapa, la población participante era de poco más del $42 \%$ de la población enérgicamente participante, entendido como el acopio y entrega diferenciada de sus residuos domésticos, lo que representó cerca de 22,000 ciudadanos.

El comportamiento territorial es el siguiente: en 23 colonias se separa y entregan los residuos diferenciados al $80 \%$, en 29 colonias al $40 \%$, mientras que en 4 colonias al 20\% (ver tabla 5).

\section{Discusión}

Con los datos reportados podemos afirmar que la escuela "cumplió su tarea", esto es, su nivel de participación como institución, alteró el involucramiento de otros actores del programa, por tanto debemos resaltar su papel en el proceso y su relevancia para llevar a cabo programas que busquen la participación de grandes segmentos de la población. Sin embargo, debemos tomar en cuenta que no fue sólo el funcionamiento de la escuela, sino la simultaneidad de acciones en los entornos fuera de ella (y dentro también).
Tabla 5

Entrega diferenciada de residuos por barrio.

\begin{tabular}{|c|c|c|}
\hline & Ahuacatal & \multirow{25}{*}{$\begin{array}{l}\text { Porcentaje de } \\
\text { la POBLACIÓN, } \\
\text { por BARRIO que } \\
\text { realiza entrega } \\
\text { DIFERENCIADA de } \\
\text { residuos }\end{array}$} \\
\hline & Bella Vista & \\
\hline & Carmen Serdán & \\
\hline & Centro & \\
\hline & Cerrito & \\
\hline 14 de Octubre & Cumbre & \\
\hline 5 de Mayo & Chapultepec & \\
\hline 5 de Octubre & El Derrumbe & \\
\hline Adolfo López Mateos & El Mirador & \\
\hline Ahuacatitla & Gregorio A. Salas & \\
\hline Benito Juárez & Guadalupe & \\
\hline Condominios Fetsse & Independencia & \\
\hline Electricistas & La Palpa & \\
\hline FETSSE & Los Ángeles & \\
\hline FOVISSTE & Los Carrizos & \\
\hline Insurgentes & Los Manantiales & \\
\hline La Aurora & Luis Donaldo Colosio & \\
\hline La Joya & Miguel Hidalgo & \\
\hline Libertad & Nuevo Monterrey & \\
\hline Lomas de Chapultepec & Ocotal & \\
\hline Los Pinos & Pajaritos & \\
\hline Magisterial & Paraíso & \\
\hline Monterrey & Piedras Pintadas & \\
\hline Petrolera & Ruiz Cortinez & \\
\hline Petrolera 2 & Santa Catarina & \\
\hline Prolongación de Juárez & Santa Cruz & Constitución \\
\hline Rio Chiquito & Santa Dora & El Potro \\
\hline Vima & Tuxpilla & La Mesita \\
\hline Zapata & Vima & Rancho Angel \\
\hline $80 \%$ & $40 \%$ & $20 \%$ \\
\hline
\end{tabular}

La simultaneidad de estrategias educativas formales, informales y no formales (las dos últimas no reportadas aquí) dieron la fuerza, expresada en la tendencia a actuar pro-ambientalmente; por tanto, es en el plano de los grupos ciudadanos y la comunidad donde podrían encontrarse los elementos complementarios, que explicarían el desenlace del proceso. Por tanto, una parte del fenómeno escapa del análisis de los dos entornos principales contenidos 
en el presente reporte (aula y escuela) y la respuesta diferenciada de los ciudadanos, a partir de su lugar de residencia, es sin duda un fuerte factor que incide sobre el comportamiento de la población escolar, tarea pendiente para los nuevos reportes.

Son tres las observaciones y la discusión sobre los entornos aula-escuela (-comunidad):

1. La EAF debe realizarse simultáneamente a las otras modalidades educativas pero, para ser más efectiva, debe contar con ímpetu, con fuerza intrínseca. Ciertamente, en una "macro-dinámica", las estrategias se potencian, empoderándose como entidad y empoderando a otros actores, en la medida que cada actor en cada entorno cumple su tarea. Sin embargo, la simultaneidad es insuficiente; la alteridad entre entornos debe garantizar una fuerza propia de los entornos intervinientes, expresada como la clara tendencia a actuar en una cierta dirección que, por ejemplo, para el entorno aula implica que la maestra(o) realice una buena sesión de clase, motivando a sus alumnos, y que estos asuman el compromiso de participación; que la escuela, además de realizar las ceremonias, periódicos murales, tarea de limpieza, etc., haga un esfuerzo para realizarlo de la mejor manera, buscando eventos significativos (las experiencias significativas, son las que no se olvidan).

2. El grado de involucramiento de un individuo y de colectivos en una tarea, es favorecido o desfavorecido en función de los entornos que lo circundan, pero sobre todo por la posición de éstos. Ciertamente, las interconexiones entre entornos son garantía de alteridad, la alteridad, en realidad, es una de las condiciones para el movimiento, el desarrollo y el cambio. Sin embargo, la posición inducida en la intervención (el entorno aula y su posición en el contexto de la escuela y la comunidad; el entorno escuela y su posición en la tarea ciudadana con respecto a los residuos; su posición en dirección al aula, para robustecer las estrategias, etc.) da el estatus (como posición) de potenciación.

3. Finalmente, podemos resaltar el papel sistémico que jugó la escuela en el programa, traducido en potencia para la generación de conductas y cogniciones proambientales, es decir, en el peso (como área emblemática del campo), que tuvo para las personas (y entornos) en relación con otras personas (y entornos).
Cada uno de los entornos es dinamizador; sin embargo, alguno de estos entornos, en este caso la escuela (como uno de los entornos vinculantes ascendente y descendentemente) desempeña el papel de "entorno eje y bisagra". Es decir, además de la posición y la fuerza, la escuela concentra la potencia en esta parte del proceso, es eje, cuando se constituye en el contexto propicio y predominante para dar sentido a las actividades realizadas en el aula (entorno específico); mientras que su papel como bisagra, le permite desempeñar un papel de vinculación y dinamización de otros entornos: aula y comunidad. La relación entre ambos no es lineal, su expresión da cuenta de múltiples acontecimientos entre e intra-entornos.

Nuestra tarea como investigadores consiste ahora en reportar los efectos puntuales de la reciprocidad en las alteridades, vinculando así la calidad con el número de acciones, es decir, evaluar la correspondencia entre las acciones llevadas a cabo por la escuela como entorno de apoyo y su relación con los momentos del proceso en el aula y fuera de la escuela, una evaluación cuali-cuanti.

\section{Referencias}

Alegre, I. (2007). Talleres comunitarios de educación ambiental para la introducción del pensamiento ambiental a nivel local. DELOS, Desarrollo Local Sostenible. 1(0).

Bermejo B. (2013). El enmascaramiento como estrategia persuasiva en la publicidad para jóvenes. Comunicar: Revista cientifica iberoamericana de comunicación y educación. 41, 157-165.

Bronfenbrenner, U. (1987). La ecología del desarrollo humano. Barcelona: Paidós.

Damergian, S. (2013). Modelos de identificación y reconstrucción cultural: Una lectura del pensamiento de Kurt Lewin. En: Problemáticas socioambientales en territorios Latinoamericanos. Guevara \& Tassara, UPAEP, México.

Durán, M., Alzate, M. y Sabucedo. (2009). La Influencia de la Norma Personal y la Teoría de la Conducta Planificada en la Separación de Residuos. Medio Ambiente y Comportamiento Humano. 10(1y2), 27-39.

Feo, M. (2010). Orientaciones básicas para el diseño de estrategias didácticas. Tendencias pedagógicas, 16, 221-236. 
Fernández, J., y Bigott, S.(2011). Alianza escuela-familiacomunidad en el Jardín de Infancia Luis Ramos Escobar: Una experiencia de desarrollo profesional docente. Revista de Investigación. 35(72), 87-114.

Flores, R. (2012). La educación ambiental: Una apuesta hacia la integración escuela-comunidad. Praxis y saber, 3 (5), 79-101.

Fuenmayor, G., y Salas, M. (2008). La escuela como centro del quehacer comunitario. Omnia, 14(1), 47-71.

Ghon, G. (2009). Educaçãonão-formal, educador (a) social e projetos sociais de inclusão social. Meta: Avaliação, 1(1), 28-43.

Giacobbe, T., y Moscoloni, B. (2009). Redes comunitarias: Un desafío para escuelas urbano-marginales. Revista Electrónica Actualidades Investigativas en Educación. 9(1), 1-33.

Golley, F. B. (1993). A History of Ecosystem Concept in Ecology: More than the Sum of the Parts. New York: Yale University Press.

Guevara, J. (2013). Programa piloto para a gestão sustentável dos residuos sólidos urbanos no município de SapeaçuBahia, Brasil. UFRB.

Guevara, J., y Rodríguez, C. (2002). Localización de actitudes pro ambientales. En: Revista de Psicología. XI (2), 92-109.

Guevara, J., y Rodríguez, C. (2003). Evaluación actitudinal y cambio de actitudes: Reporte de una investigación aplicada focalizada en la basura. Revista Chilena de Psicología, 24(1).

Lewin, K. (1936). A Dynamic Theory of Personality, NY: McGraw Hill. Trad. Cast.: Dinámica de la personalidad, Madrid, Morata, S.A., 1973.

Morin, E. (2008). O Método. 1. A natureza da natureza, Trad. Ilana Heineberg. Porto Alegre, 2a edición.

OPS (2005). Informe de la evaluación regional de los servicios de manejo de Residuos Sólidos Municipales en América Latina y el Caribe. Washington, D.C. http://www. bvsde.ops-oms.org/bvsars/fulltext/informe/introduc.pdf
OPS (2006). Diagnóstico de la situación del manejo de residuos sólidos municipales en América Latina y el Caribe: http://www.bvsde.paho.org/eswww/fulltext/ resisoli/dsm/dsm.html

Pontes, C. (2013). El pensamiento ecológico contemporáneo: La ciencia de los ecosistemas. En: Problemáticas socioambientales en territorios Latinoamericanos. Guevara \& Tassara, UPAEP, México.

Quintanar, F. (2011). Actitudes orientadas a basura y servicio de recolección de basura. En: J. Guevara, Segundo Informe al CONACYT del "Proyecto Ejecutivo para el manejo integral de los desechos sólidos domésticos en Huauchinango y estudio de caracterización de desechos en cinco zonas urbanas de la Sierra Norte de Puebla".

Ramírez, P. (2008). Educación ambiental e integración escuela comunidad. Geoenseñanza, 13(1), 105-114.

Rojas, H., y Lara M. (2004). Enfoques de análisis de los contextos educativos. Diálogos Educativos, (8).

Stefan, D. (2010). Congresso Internacional de Negócios da Indústria da Reciclagem, (28/09/2010) São Paulo (Conferencia que abrió el evento). Fuente: Vinicius Konchinski/ Agência Brasil, Redação do Numa 2020 Sustentável-Resíduos Sólidos Urbanos. Disponible en: http://2020sustentavelresiduossolidosurban os.blogspot.com.br/2011/07/gestao-de-residuossolidos-no-brasil-no.html

Torres, R. M. (2001). Participación Ciudadana y Educación, Una Mirada Amplia y 20 Experiencias en América Latina. Documento encargado por la Unidad de Desarrollo Social y Educación (UDSE) de la OEA para su presentación en la Segunda Reunión de Ministros de Educación del Consejo Interamericano para el Desarrollo Integral - CIDI (Punta del Este, Uruguay, 24-25 Septiembre, 2001).

Torres, M., Mora, G., Garzón, V., y Ceballos, B. (2013). Desarrollo de competencias científicas a través de la aplicación de estrategias didácticas alternativas. Un enfoque a través de la enseñanza de las ciencias naturales. Tendencias, 14(1), 187-215. 
